\title{
Ethylene polymerization in supercritical carbon dioxide with binuclear nickel(II) catalysts
}

\author{
Damien Guironnet, Tobias Friedberger and Stefan Mecking*
}

\author{
Received 30th June 2009, Accepted Ist September 2009 \\ First published as an Advance Article on the web 21st September 2009 \\ DOI: $10.1039 / \mathrm{b} 912883 \mathrm{~b}$
}

\begin{abstract}
A series of new, highly fluorinated neutral $\left(\kappa^{2}-N, O\right)$ chelated $\mathrm{Ni}$ (II) binuclear complexes based on salicylaldimines bridged in $p$-position of the $N$-aryl group were prepared. The complexes are single-component catalyst precursors for ethylene polymerization in supercritical carbon dioxide and toluene. Solubility of the catalyst precursors in supercritical carbon dioxide is effected by a large number of up to 18 trifluoromethyl groups per molecule. Semicrystalline polyethylene with a low degree of branching is formed ( $c a .10$ branches $/ 1000$ carbon atoms). Polymer microstructures are independent of the nature of the bridging moiety, while stability of the catalysts appears to differ.
\end{abstract}

\section{Introduction}

Polymerization of olefins catalyzed by complexes of $\mathrm{d}^{8}$ metals (late transition metals) has been investigated intensively recently. ${ }^{1}$ In comparison to their early transition metal counterparts, they are much more tolerant towards functional groups in the substrates ${ }^{2 a, b}$ or reaction media. ${ }^{2 c}$ Thus, ethylene and 1-olefins can be copolymerized with electron-deficient polar vinyl monomers, such as e.g. acrylates ${ }^{3}$ or even acrylonitrile, ${ }^{4}$ and multiple insertion of acrylates affords low molecular weight polyacrylate via an insertion mechanism., ${ }^{3,6}$ Polymerization of ethylene and 1-olefins can be carried out in oxygenated reaction media such as water ${ }^{7}$ or dense carbon dioxide. ${ }^{8}$

Dense carbon dioxide, that is liquid or supercritical $\mathrm{CO}_{2}$ $\left(\mathrm{seCO}_{2}\right)$, possesses unique properties, such as the possibility of variation of its density and solvent properties over a wide range. ${ }^{9}$ In polymerization processes and polymer processing, dense carbon dioxide can be useful as a solvent or suspension medium. ${ }^{96,10}$ It can be removed conveniently by variation of the pressure, resulting in a dry polymer powder. While free-radical polymerization in dense $\mathrm{CO}_{2}$ has been studied most intensely, " various examples of coordination polymerization have been reported. ${ }^{12-14}$ Ethylene polymerization has been studied in $\mathrm{scCO}_{2}$ with cationic palladium diimine complexes ${ }^{\mathrm{san}-\mathrm{c}}$ as well as with neutral $\left(\mathrm{\kappa}^{2}-N, O\right)$ nickel complexes. ${ }^{\text {st,e }}$

Current developments of late transition metal catalyzed olefin polymerization have resulted in a renewed ${ }^{13}$ interest in neutral Ni(II) complexes. ${ }^{16}$ This class of catalysts appears to be particularly suited for polar (protic) reaction media. ${ }^{7,17}$ Substantial effort is devoted to the finding of catalysts with increased polymerization productivity. One approach studied are binuclear complexes, which often polymerize with higher activities than their mononuclear analogues. ${ }^{18}$ The origin of this increased reactivity is illunderstood, cooperative effects have been suggested to operate in certain cases. ${ }^{18 \mathrm{~h}}$ Another aim of catalyst development is an appropriate solubility in the specific reaction media. In aqueous

Chair of Chemical Materials Science, Dept. of Chemistry, University of Konstanz, 78464, Konstanz, Germany. E-mail: stefan.mecking@unikonstanz.de; Fax: $+497531885152 ;$ Tel: +497531885151 emulsion systems this can be crucial for the control of nanoparticle size and structure. ${ }^{7 \mathrm{~d},}$ Concerning reactions in $\mathrm{CO}_{2}$, tailoring of the catalyst precursors to provide solubility in the reaction medium is required.

We now report binuclear salicylaldiminato Ni(II) methyl complexes soluble in dense $\mathrm{CO}_{2}$ and their polymerization properties.

\section{Results and discussion}

\section{Synthesis of ligands and complexes}

Most commonly, solubility of organic compounds and of catalysts, in particular, in dense $\mathrm{CO}_{2}$ is brought about by longer perfluoroalkyl groups, as exemplified by the commercially available tris[3(perfiuorooctyl)phenyl]phosphine. Introduction of such perfluoro alkyl moieties is associated with considerable synthetic effort and specific work up procedures. A convenient alternative can be the introduction of a sufficiently large number of trifluoromethyl groups. It is worth noting that such electron withdrawing groups will also modify the electrophilicity of the metal center and consequently catalytic properties (vide infra). The binuclear complexes reported here are based on 3 differently bridged salicyladimine ligands, 3a (benzidine bridge), 3b (methylene bridge) and $\mathbf{3 c}$ (hexafluoroisopropylidene bridge).

Dianilines $1 \mathbf{a}$ (benzidine scaffold) and $\mathbf{1 b}$ (methylene bridge) were prepared according to known procedures. ${ }^{185}$ Ic (hexafluoroisopropylidene bridge) was obtained analogously by selective ortho-bromination of $4,4^{\prime}$-diamino diphenyl $1,1,1,3,3,3$ hexafluoropropane, and subsequent Suzuki coupling with 3,5bis(trifuoromethyl)phenyl boronic acid in good yield (overall yield: $63 \%$ ). Condensation of the dianilines $1 \mathrm{a}-\mathrm{c}$ with $3,5-$ bis[3,5-bis(trifluoromethyl)phenyl] salicylaldehyde in toluene under Dean-Starck conditions afforded the novel salicylaldimines 2a-c (Scheme 1). The crude products obtained upon solvent evaporation were washed with methanol. The identity and purity of the dianilines and di(salicylaldimines) were established by ' $\mathrm{H}$, ${ }^{13} \mathrm{C}$ NMR spectroscopy, mass spectrometry and elemental analysis (see Experimental).

Reaction of the respective di(salicylaldimines) with 3 equivalents of $\left[(\text { pyridine })_{2} \mathrm{Ni}\left(\mathrm{CH}_{3}\right)_{2}\right]$ in cold diethylether suspension 


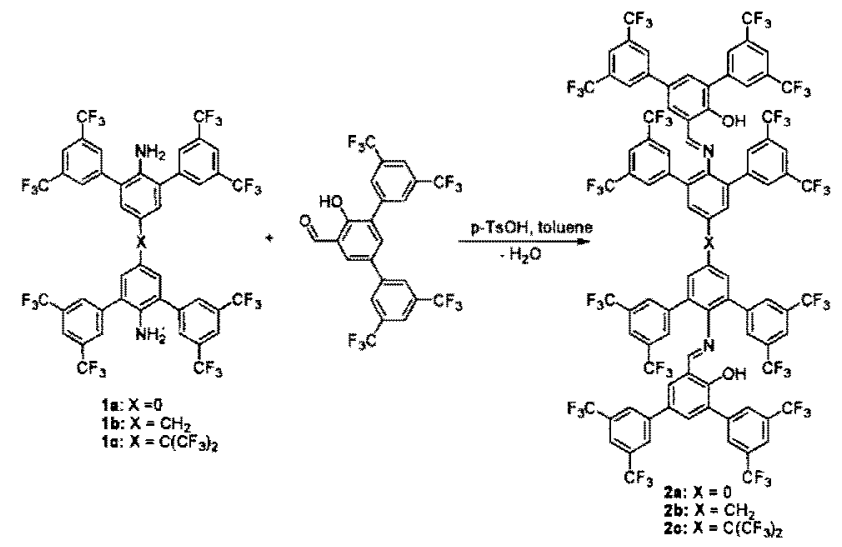

Scheme 1 Preparation of binuclear salicylaldimine ligands.

was found to be a viable route for the preparation of complexes 3a-c (Scheme 2). The relatively poor solubility of the ligand in diethylether slows down the reaction. Consumption of the starting material could be followed by a colour change (from yellow to dark red), and by the evolution of methane. Decomposed excess Ni(II) starting material was removed by filtration.

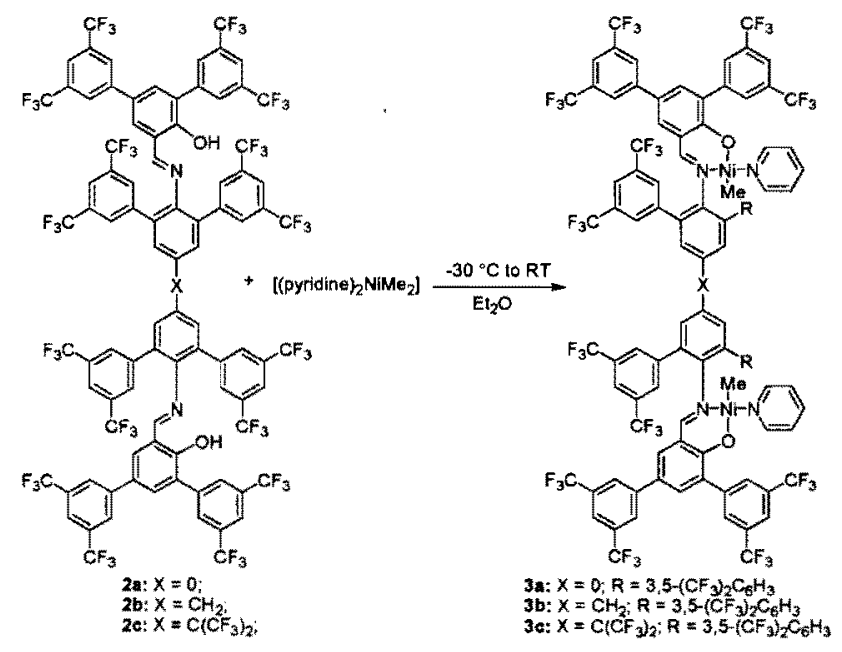

Scheme 2 Preparation of $\left(\kappa^{2}-N, O\right)$ salicylaldiminato nickel-methyl pyridine complexes.

All complexes are diamagnetic in solution as evidenced by sharp resonances in the ${ }^{1} \mathrm{H}$ and ${ }^{13} \mathrm{C}$ NMR spectra. This suggests a square planar coordination geometry of the nickel center, as reported previously for other $\mathrm{Ni}(\mathrm{II})$-Me salicylaldiminato complexes. ${ }^{168,186,19}$ Most characteristic are the high field nickel methyl resonances around $-1.0 \mathrm{ppm}$ in the ${ }^{1} \mathrm{H}$ NMR and $-8 \mathrm{ppm}$ in the ${ }^{13} \mathrm{C}$ NMR spectra. This unique resonance confirms that both metal centers are magnetically identical in the binuclear structure, as expected.

\section{Catalytic ethylene polymerization with binuclear $\left(\kappa^{2}-N, O\right)$ salicylaldiminato $\mathrm{Ni}(\mathrm{II})$ methyl pyridine complexes}

Polymerization studies were performed in toluene as a reaction medium (Table 1). In this case monitoring of the polymerization via mass-flow meters, not possible with the set-up for polymerization in supercritical fluids, gives insights on catalyst stability. The mononuclear analogue $4^{\text {8d }}$ was studied as a catalyst precursor for comparison (entries 1-7 and 1-8).

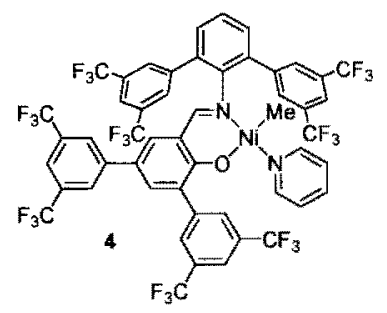

The novel catalyst precursors are active for ethylene polymerization at $50{ }^{\circ} \mathrm{C}$ under 40 bar ethylene pressure in toluene. Independently of the bridge, activities observed were on the same order as for the mononuclear analogue $\left(3 \times 10^{4} \mathrm{TO} \mathrm{h}^{-1}\right.$ average in a 30 min experiment). At a polymerization temperature of $70^{\circ} \mathrm{C}$, higher polymerization rates were observed (Table 1). This is likely associated with an enhanced dissociation of the pyridine donor. At this temperature, the nature of the bridge does have a clear effect on catalyst stability. Complexes $\mathbf{3 a , b}$ as well as $\mathbf{4}$ decomposed rapidly. By contrast, $3 c$ consumed ethylene steadily over the $30 \mathrm{~min}$ duration of the experiment.

The polymers obtained are semicrystalline $(\chi-50 \%)$ with a melt peak temperature in the range from 105 to $122^{\circ} \mathrm{C}$. A relatively low number of methyl branches was detected by ${ }^{13} \mathrm{C}$ NMR spectroscopy ( 15 per 1000 carbon atoms) along with very few ethyl branches ( 2 per 1000 carbon atoms). Note that these branches result from 'chain walking", ${ }^{20}$ commonly observed with late transition metal catalysts. In detail, the lowest branching and highest polymer molecular weight was observed for $\mathbf{3 c}$, which can be related to the electron-withdrawing character of the hexafluoropropylidine bridge. An increased electrophilicity is

Table 1 Polymerization in toluene

\begin{tabular}{|c|c|c|c|c|c|c|c|c|c|}
\hline Run & Complex & $T /{ }^{*} \mathrm{C}$ & $\operatorname{TOF}^{c} 10^{3} / \mathrm{h}^{-1}$ & $M_{1 \mathrm{~d}}^{d} 10^{3} / \mathrm{g} \mathrm{mol}^{-1}$ & $M_{\mathrm{w}} / M_{\mathrm{a}}{ }^{d}$ & $T_{m} / f^{\prime} \mathrm{C}$ & $x^{*}(\%)$ & $N_{\mathrm{Me}} / 1000 \mathrm{C}^{f}$ & $N_{\mathrm{Kak}} / 1000 \mathrm{C}$ \\
\hline $1-1$ & $3 \mathbf{a}$ & 50 & 18 & 23.0 & 2.4 & 115 & 50 & 14 & $<1$ \\
\hline $1-2$ & $3 \mathbf{a}$ & 70 & 64 & 3.8 & 3.1 & 104 & 45 & 19 & 2 \\
\hline $1-3$ & $3 \mathbf{b}$ & 50 & 20 & 25.1 & 2.8 & 119 & 51 & 11 & $<1$ \\
\hline $1.4^{\circ}$ & $\mathbf{3 b}$ & 70 & 25 & 2.6 & $3.9^{\mathrm{g}}$ & 110 & 47 & 15 & 2 \\
\hline $1-5$ & $3 \mathrm{c}$ & 50 & 29 & 38.9 & 2.6 & 122 & 54 & 9 & $<\overline{1}$ \\
\hline $1-6$ & $3 c$ & 70 & 91 & 12.7 & 2.1 & 115 & 54 & 14 & $<1$ \\
\hline $1.77^{\circ}$ & 4 & 50 & 32. & 14.3 & 2.4 & 110 & 48 & 17 & 2 \\
\hline $1-8^{b}$ & 4 & 70 & 70 & 8.3 & 2.2 & 105 & 46 & 19 & 2 \\
\hline
\end{tabular}

${ }^{a}$ Reaction conditions: $100 \mathrm{~mL}$ of toluene, $p$ (ethylene) $=40$ bar, $t=30 \mathrm{~min}, n(\mathrm{Ni})=10 \mu \mathrm{mol} .{ }^{b} n(\mathrm{Ni})=5 \mu \mathrm{mol} .{ }^{c} \mathrm{TOF}$ in mol $\left(\mathrm{C}_{2} \mathrm{H}_{4}\right) \mathrm{mol}(\mathrm{Ni})^{-1} \mathrm{~h}^{-1}$. ${ }^{a}$ Determined by GPC. "Determined by DSC from second heat. 'Determined by ${ }^{13} \mathrm{C}$ NMR. "Bimodal distribution. 
Table 2 Polymerization in $\mathrm{seCO}_{2}{ }^{*}$

\begin{tabular}{|c|c|c|c|c|c|c|c|c|c|}
\hline Run & Cat. & $T /^{\circ} \mathrm{C}$ & TOFe $10^{3} / \mathrm{h}^{-1}$ & $M_{\mathrm{n}}^{f} 10^{3} / \mathrm{g} \mathrm{mol}^{-1}$ & $M_{w} / M_{n}^{f}$ & $T_{\mathrm{m}} \times /{ }^{\circ} \mathrm{C}$ & $\chi^{8}(\%)$ & $N_{\mathrm{Me}} / 1000 \mathrm{C}^{h}$ & $N_{k t} / 1000 C^{*}$ \\
\hline $2-1^{\circ}$ & $3 \mathbf{a}$ & 50 & 2.7 & 15.3 & 2.3 & 121 & 48 & 14 & $<1$ \\
\hline $2-2^{b}$ & $3 \mathbf{b}$ & 50 & 4.3 & 29.2 & 2.5 & 119 & 47 & n.d. & n.d. \\
\hline $2-3$ & 3b & 70 & 10.4 & 1.8 & 3.8 & 98 & 36 & 17 & 2 \\
\hline $2-4^{b}$ & $\mathbf{3} \mathbf{b}^{c}$ & 70 & 15.1 & 12.0 & 2.1 & 99 & 36 & 17 & 2 \\
\hline $2-5^{b}$ & $3 c$ & 50 & 3.4 & 26.1 & 2.3 & 124 & 54 & 9 & $<\overline{1}$ \\
\hline $2-6$ & $3 \mathrm{c}$ & 70 & 8.9 & 2.3 & 4.6 & 105 & 43 & 14 & 1 \\
\hline 2.7 & $3 c^{c}$ & 70 & 14.3 & 2.7 & 3.4 & 105 & 43 & 14 & 2 \\
\hline $2-8$ & $4^{d}$ & 50 & 5.7 & 33.3 & 2.1 & 123 & 49 & 5 & $<\overline{1}$ \\
\hline 2.9 & $4^{d}$ & 70 & 10.9 & 2.9 & 3.4 & 97 & 40 & 19 & 2 \\
\hline
\end{tabular}

${ }^{a}$ Reaction conditions: $n(\mathrm{Ni})=10 \mu \mathrm{mol}, t=30 \mathrm{~min}, m\left(\mathrm{CO}_{2}\right)=45.5 \mathrm{~g}, m\left(\mathrm{C}_{2} \mathrm{H}_{4}\right)=6 \mathrm{~g} .{ }^{b} m\left(\mathrm{C}_{2} \mathrm{H}_{4}\right)=2 \mathrm{~g} \cdot{ }^{c} n(\mathrm{Ni})=5 \mu \mathrm{mol} .{ }^{d} n(\mathrm{Ni})=2.5 \mu \mathrm{mol} .{ }^{c} \mathrm{TOF}$ in $10^{3} \mathrm{~mol}\left(\mathrm{C}_{2} \mathrm{H}_{4}\right) \mathrm{mol}\left(\mathrm{Ni}^{-1} \mathrm{~h}^{-1} \cdot{ }^{\prime}\right.$ Determined by GPC. Determined by DSC from second heating. ${ }^{\text {" Determined by }}{ }^{13} \mathrm{NMR}$.

known to reduce the tendency for $\beta-H$ elimination, a key step of both branch formation and chain transfer. ${ }^{16 \mathrm{~m}}$

Polymerizations in $\mathrm{scCO}_{2}$ were performed in a high pressure reactor equipped with sapphire windows, which enable a visual monitoring of the reaction. Catalyst precursors were found to dissolve at a moderate pressure in the supercritical fuid $\left(50^{\circ} \mathrm{C}, 35\right.$ $\mathrm{MPa}$ ), to form a bright yellow-orange solution. After addition of ethylene, an immediate onset of turbidity was observed due to the precipitation of the polyethylene formed. Upon careful venting of the reactor after the desired polymerization time, the polymer was obtained directly as a dry powder. The binuclear complexes polymerized ethylene in $\mathrm{scCO}_{2}$ with up to $10^{4} \mathrm{TO} \mathrm{h}^{-1}$ at $70^{\circ} \mathrm{C}$ (Table 2). As previously observed for catalytic polymerization of ethylene in dense carbon dioxide with other salicylaldimine complexes, ${ }^{8 d}$ productivities are significantly decreased by comparison to polymerization in organic hydrocarbon solvent. A possible explanation for these lower activities is an enclosure of the intact catalyst in the precipitated polymer, which will also be less swollen with solvent by comparison to toluene as a reaction medium. Polymers prepared at $50{ }^{\circ} \mathrm{C}$ possess similar molecular weights, degrees of branching, and melting properties as observed in toluene. However, the reduction of polymer molecular weight with increased reaction temperature is more pronounced in $\mathrm{scCO}_{2}$ than the organic solvent. It can be speculated that a coordinating nature of $\mathrm{CO}_{2}$ contributes to chain transfer via an associative mechanism. ${ }^{16 \mathrm{~b}}$

\section{Summary and conclusion}

Three novel highly fluorinated binuclear $\mathrm{Ni}(\mathrm{II})$ methyl pyridine complexes of di(salicyladimines) bridged in the p-position of the $N$-aryl moiety were prepared and characterized. These compounds were found to be single-component catalyst precursors for the polymerization of ethylene in supercritical carbon dioxide as a reaction medium. A polymerization temperature of $70^{\circ} \mathrm{C}$ resulted in higher polymerization activities than at $50^{\circ} \mathrm{C}$, likely due to enhanced pyridine dissociation, however at the expense of polymer molecular weights, which was particularly pronounced in $\mathrm{scCO}_{2}$ by comparison to an organic reaction medium. Semicrystalline polyethylene with a rather moderate degree of branching and moderate molecular weight is formed invariable of the catalyst structure. However, the bridging moiety does influence catalyst stability over time, as observed for polymerization in toluene.

\section{Experimental}

\section{Materials and general considerations}

Unless noted otherwise, all manipulations of metal complexes were carried out under an inert atmosphere using standard glovebox or Schlenk techniques. All glassware was flamedried under vacuum before use. Toluene and benzene were distilled from sodium, diethylether from sodium/benzophenone ketyl under argon. Ethylene (99.95\%) and carbon dioxide (99.9995\%) supplied by Praxair and Air Liquide, respectively, were used as received. All other solvents were commercial grade. 4,4'-diamino diphenyl 1,1,1,3,3,3-hexafluoropropane was purchased from Aldrich. [(pyridine $)_{2} \mathrm{Ni}\left(\mathrm{CH}_{3}\right)_{2}$ ] was synthesized by a modified literature procedure ${ }^{21}$ and stored at $-30{ }^{\circ} \mathrm{C}$ in a glovebox. 3,5-bis[3,5-bis(trifluoromethyl)phenyl] salicylaldehyde, ${ }^{22} \quad 3,3^{\prime}, 5,5^{\prime}$-tetrakis(3,5-bis(trifluoromethyl)-phenyl)4,4'-diaminodiphenylmethane, ${ }^{18 \mathrm{~d}} \quad 3,3^{\prime}, 5,5^{\prime}$-tetrakis-[3,5bis(trifluoromethyl)phenyl]benzidine, ${ }^{18 d}$ and complex $4^{22}$ were prepared according to reported procedures.

NMR spectra were recorded on a Varian Unity INOVA 400 or on a Bruker Avance DRX 600 spectrometer. ${ }^{1} \mathrm{H}$ and ${ }^{13} \mathrm{C}$ NMR chemical shifts were referenced to the solvent signal. NMR assignments were confirmed by ${ }^{1} \mathrm{H},{ }^{\prime} \mathrm{H}$ gCOSY, ${ }^{1} \mathrm{H},{ }^{13} \mathrm{C}$ gHSQC and ${ }^{1} \mathrm{H},{ }^{13} \mathrm{C}$ gHMBC experiments. Elemental analyses were performed up to $950^{\circ} \mathrm{C}$ on an Elementar Vario EL. High-temperature NMR measurements of polyethylenes were performed in $1,1,2,2$ tetrachloroethane- $\mathrm{d}_{2}$ at $130^{\circ} \mathrm{C}$. Size exclusion chromatography (GPC) was carried out in 1,2,4-trichlorobenzene at $160^{\circ} \mathrm{C}$ at a flow rate of $1 \mathrm{~mL} \mathrm{~min}{ }^{-1}$ on a Polymer Laboratories 220 instrument equipped with Olexis columns with differential refractive index, viscosity-and light scattering- $\left(15^{\circ}\right.$ and $\left.90^{\circ}\right)$ detectors. Data reported were determined $v s$. polyethylene standards. Differential scanning calorimetry (DSC) was performed on a Netzsch Phoenix $204 \mathrm{Fl}$ at a heating rate of $10 \mathrm{~K} \mathrm{~min}^{-1}$. DSC data reported are from second heating cycles. Polymer crystallinity was calculated based on a melt enthalpy of $293 \mathrm{~J} \mathrm{~g}^{-1}$ for $100 \%$ crystalline polyethylene.

\section{3,3 , $, 5,5$, '-Tetrabromo 4,4'-diamino diphenyl $1,1,1,3,3,3$-hexafluoropropane}

To a suspension of tetra- $n$-butylammonium tribromide $(17.31 \mathrm{~g}$, $36 \mathrm{mmol}$ ) and calcium carbonate $(3.59 \mathrm{~g}, 36 \mathrm{mmol})$ in $70 \mathrm{~mL}$ of methanol was added a solution of 4,4'-diamino diphenyl $1,1,1,3,3,3$-hexafluoropropane $(3.00 \mathrm{~g}, 9 \mathrm{mmol})$ in $30 \mathrm{~mL}$ of 
methanol, and the mixture was stirred at room temperature. The colour changed from an initial orange to light green. After $12 \mathrm{~h}$, the solvent was removed in vacuo, and the residue was extracted with hot toluene on a frit. The extract was concentrated to dryness and washed twice each with water and methanol. The resulting crude produet was recrystallized from toluene as brown large needles. ${ }^{1} \mathrm{H}$ NMR (400 MHz, $\left.\mathrm{CDCl}_{3}\right): \delta_{\mathrm{H}}(\mathrm{ppm}) ; 7.36(\mathrm{~s}, 2 \mathrm{H}, 3 \mathrm{H}), 4.76(\mathrm{~s}, 2$ $\left.\mathrm{H}, \mathrm{N} H_{2}\right) .{ }^{13} \mathrm{C} \mathrm{NMR}\left(101 \mathrm{MHz}, \mathrm{CDCl}_{3}\right) \delta_{\mathrm{C}}(\mathrm{ppm}) ; 143.10,133.32$ (C3), $123.97\left(\mathrm{q},{ }^{1} J_{\mathrm{CF}}=287 \mathrm{~Hz}\right), 123.24,108.13,62.60$ (septet, $\left.{ }^{2} J_{\mathrm{CF}}=26.8, \mathrm{C} 5\right)$. Anal. calcd for $\mathrm{C}_{15} \mathrm{H}_{8} \mathrm{Br}_{4} \mathrm{~F}_{6} \mathrm{~N}_{2}(M=649.24 \mathrm{~g}$ $\mathrm{mol}^{-1}$ ): C 27.72, H 1.24, N 4.32. Found: C 28.74, H 1.44, N 4.17.<smiles>Nc1c(Br)cc(C(c2cc(Br)c(N)c(Br)c2)(C(F)(F)F)C(F)(F)F)cc1Br</smiles>

\section{3,3 $3^{\prime}, 5,5^{\prime}$-Tetrakis(3,5-bis(triftuoromethyl)phenyl) 4,4'-diamino diphenyl 1,1,1,3,3,3-hexafluoropropane}

To a mixture of $3,3^{\prime}, 5,5$, $^{\prime}$-tetrabromo $4,4^{\prime}$-diamino diphenyl $1,1,1,3,3,3$-hexafluoropropane $(0.86 \mathrm{mmol}, 0.56 \mathrm{~g}), 5$ equiv. of 3,5-bis(trifluoromethyl)phenyl boronic acid $(4.28 \mathrm{mmol}, 1.10 \mathrm{~g}$ ), $2.5 \mathrm{~mol} \%\left[\mathrm{Pd}(\mathrm{dba})_{2}\right](0.02 \mathrm{mmol}, 12 \mathrm{mg})$, and $5 \mathrm{~mol} \%$ triphenyl phosphine $(0.04 \mathrm{mmol}, 11 \mathrm{mg})$ were added $20 \mathrm{~mL}$ of toluene, $5 \mathrm{~mL}$ of ethanol and $10 \mathrm{~mL}$ of a $2 \mathrm{M} \mathrm{Na}_{2} \mathrm{CO}_{3}$ aqueous solution in a Schlenk flask under protective atmosphere. The suspension was heated with vigorous stirring to $90^{\circ} \mathrm{C}$ for $24 \mathrm{~h}$. The resulting biphasic mixture was stirred for $30 \mathrm{~min}$ under air (resulting in formation of palladium black) and poured into a separatory funnel, water and diethyl ether were added until all salts and organic material dissolved. The aqueous phase was extracted with additional $2 \times 25 \mathrm{~mL}$ of diethyl ether. The organic phases were combined, and filtrated through a plug of silica to remove $\mathrm{Pd}$ black. The solvents were removed under vacuum. The product was dissolved in hot hexane and recrystallised slowly out of this solution. Yield: $87 \%$. ' $\mathrm{H}$ NMR $\left(400 \mathrm{MHz}, \mathrm{CDCl}_{3}\right): \delta_{\mathrm{H}}(\mathrm{ppm})$; $7.84(\mathrm{~s}, 4 \mathrm{H}, 11-\mathrm{H}), 7.81(\mathrm{~s}, 8 \mathrm{H}, 8-\mathrm{H}), 7.18(\mathrm{~s}, 4 \mathrm{H}, 3-\mathrm{H}), 3.77$ (s, $\left.4 \mathrm{H}, \mathrm{NH} \mathrm{H}_{2}\right) .{ }^{13} \mathrm{C} \mathrm{NMR}\left(101 \mathrm{MHz}, \mathrm{CDCl}_{3}\right) \delta_{\mathrm{C}}(\mathrm{ppm}): 141.66$ (s, C7), $140.56(\mathrm{~s}, \mathrm{C}), 132.75(\mathrm{~s}, \mathrm{C} 3), 133.06\left(\mathrm{q},{ }^{2} J_{\mathrm{CF}}=33.7, \mathrm{C} 9\right)$, $129.76(\mathrm{~s}, \mathrm{C} 8), 125.30(\mathrm{~s}, \mathrm{C} 4), 123.40(\mathrm{~s}, \mathrm{C} 2), 124.44\left(\mathrm{q},{ }^{1} J_{\mathrm{CF}}=\right.$ $287.5, \mathrm{C} 10)\left(\mathrm{q},{ }^{1} J_{\mathrm{CF}}=273.3, \mathrm{C} 6\right), 122.38$ (septet, ${ }^{3} J_{\mathrm{CF}}=3.5, \mathrm{Cl} 1$ ), 63.82 (septet, $\left.{ }^{2} J_{C F}=26.0, \mathrm{C} 5\right)$. Anal. calcd for $\mathrm{C}_{\mathrm{d} 7} \mathrm{H}_{20} \mathrm{~F}_{30} \mathrm{~N}_{2}(M=$ $\left.1182.63 \mathrm{~g} \mathrm{~mol}^{-1}\right): \mathrm{C} 47.73, \mathrm{H} 1.70, \mathrm{~N} 2.37$. Found: $\mathrm{C} 48.31, \mathrm{H} 2.31$, N 2.32 .

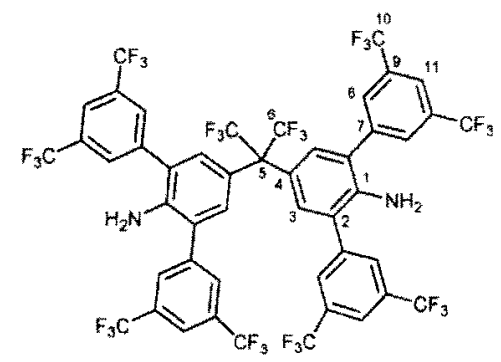

General procedure for condensation of anilines with 3,5-(bis(trifluoromethyl)phenyl) salicylaldehyde.

In a flask equipped with a Dean-Stark condenser, the aniline, the aldehyde (1.1 equiv, ) and a catalytic amount of $p$-toluenesulfonic acid were dissolved in $100 \mathrm{~mL}$ of toluene. The mixture was degassed by several cycles of evacuating and back filling with inert atmosphere. The mixture was stirred at $80^{\circ} \mathrm{C}$, and the pressure was reduced until the solvent boiled. The mixture was kept at $80^{\circ} \mathrm{C}$ for $12 \mathrm{~h}$. The reaction mixture was concentrated, and to the brown viscous residue was added $20 \mathrm{~mL}$ of methanol to afford the precipitation of the desire product as a yellowish powder.

\section{$I\left\{\left(2,6-\left(3,5-\left(\mathrm{CF}_{3}\right)_{2} \mathrm{C}_{6} \mathrm{H}_{3}\right)_{2}-4-\mathrm{y}\right]-\mathrm{C}_{6} \mathrm{H}_{2}\right)-\mathrm{N}=\mathrm{C}(\mathrm{H})-(3,5-(3,5-$ $\left.\left.\left.\left(\mathrm{CF}_{3}\right)_{2} \mathrm{C}_{6} \mathrm{H}_{3}\right) 2-2-(\mathrm{OH})-\mathrm{C}_{6} \mathrm{H}_{2}\right)\right\}_{2} \mathrm{l}(2 \mathrm{a})$}

Yield: $56 \%$, 'H NMR $\left(400 \mathrm{MHz}, \mathrm{CDCl}_{3}\right): \delta_{\mathrm{H}}(\mathrm{ppm}): 12.85(2 \mathrm{H}$, s), $8.21(2 \mathrm{H}, \mathrm{s}), 8.07(4 \mathrm{H}, \mathrm{s}), 8.03(8 \mathrm{H}, \mathrm{s}), 7.94(2 \mathrm{H}, \mathrm{s}), 7.92(4 \mathrm{H}$, s), $7.90(8 \mathrm{H}, \mathrm{s}), 7.87(2 \mathrm{H}, \mathrm{s}), 7.70(2 \mathrm{H}, \mathrm{s}), 7.28(2 \mathrm{H}, \mathrm{s})$. MALDITOF-MS: $m / z 2090.2\left([\mathrm{M}+\mathrm{H}]^{+}\right)$Anal. calcd for $\mathrm{C}_{90} \mathrm{H}_{36} \mathrm{~F}_{48} \mathrm{~N}_{2} \mathrm{O}_{2}$ $\left(M=2089.2 \mathrm{~g} \mathrm{~mol}^{-1}\right): \mathrm{C} 51.74, \mathrm{H} 1.74, \mathrm{~F} 43.65, \mathrm{~N} 1.34$. Found: C $50.58, \mathrm{H} 2.34, \mathrm{~F} 43.68, \mathrm{~N} 1.74$.

\section{$\left[\mathrm{CH}_{2}\left\{\left(\mathbf{2 , 6} 6-\left(3,5-\left(\mathrm{CF}_{3}\right)_{2} \mathrm{C}_{6} \mathrm{H}_{3}\right) 2-4-\mathrm{yl}-\mathrm{C}_{6} \mathrm{H}_{2}\right)-\mathrm{N}=\mathrm{C}(\mathrm{H})-(3,5-(3,5-\right.\right.$ $\left.\left.\left.\left(\mathrm{CF}_{3}\right)_{2} \mathrm{C}_{6} \mathrm{H}_{3}\right)_{2}-2-(\mathrm{OH})-\mathrm{C}_{6} \mathrm{H}_{2}\right)\right\}_{2} \mathrm{l}(2 \mathrm{~b})$}

'H NMR (400 MHz, $\mathrm{CDCl}_{3}$ ): $\delta_{\mathrm{H}}(\mathrm{ppm}) ; 12.72$ (br. s, $\left.2 \mathrm{H}\right) 8.13$ (s, $2 \mathrm{H}), 7.98(\mathrm{~s}, 4 \mathrm{H}), 7.87(\mathrm{~s}, 10 \mathrm{H}), 7.81(\mathrm{~s}, 10 \mathrm{H}), 7.59\left(\mathrm{~d},{ }^{4} J_{\mathrm{HH}}=\right.$ $2.3,2 \mathrm{H}), 7.45(\mathrm{~s}, 4 \mathrm{H}), 7.12\left(\mathrm{~d},{ }^{4} J_{\mathrm{HH}}=2.3,2 \mathrm{H}\right), 4.32(\mathrm{~s}, 2 \mathrm{H})$. ${ }^{13} \mathrm{C}$ NMR (101 MHz, $\left.\mathrm{CDCl}_{3}\right) \delta_{\mathrm{C}}(\mathrm{ppm}): 169.54,158.70,143.88$, $141.44,140.60,139.43,138.37,133.63,132.93,132.84\left(\mathrm{q},{ }^{2} J_{\mathrm{CF}}=\right.$ $33), 132.43\left(\mathrm{q},{ }^{2} J_{\mathrm{CF}}=33\right), 131.87,131.47\left(\mathrm{q},{ }^{2} J_{\mathrm{CF}}=33\right), 131.45$, $130.40,130.12,130.09,129.68,128.69,126.90,123.56\left(\mathrm{q},{ }^{1} J_{\mathrm{CF}}=\right.$ $273), 123.40\left(\mathrm{q},{ }^{1} J_{\mathrm{CF}}=273\right), 123.22\left(\mathrm{q},{ }^{1} J_{\mathrm{CF}}=273\right), 121.85,121.58$, 118.85, 40.85. MALDI-TOF-MS: $m / z$ 2104.2 $\left(\left[\mathbf{M}+\mathbf{H}^{+}\right)\right.$Anal. calcd for $\mathrm{C}_{91} \mathrm{H}_{38} \mathrm{~F}_{48} \mathrm{~N}_{2} \mathrm{O}_{2}\left(M=2103.2 \mathrm{~g} \mathrm{~mol}^{-1}\right): \mathrm{C} 51.97, \mathrm{H} 1.82, \mathrm{~F}$ 43.36, N 1.33. Found: C 50.97, H 1.82, F 43.40, N 1.32.

\section{$\left[\mathrm{C}\left(\mathrm{CF}_{3}\right)_{2}\left\{\left(2,6-\left(3,5-\left(\mathrm{CF}_{3}\right)_{2} \mathrm{C}_{6} \mathrm{H}_{3}\right)_{2}-4-\mathrm{yl}-\mathrm{C}_{6} \mathrm{H}_{2}\right)-\mathrm{N}=\mathrm{C}(\mathrm{H})-(3,5-(3,5-\right.\right.$ $\left.\left.\left.\left(\mathrm{CF}_{3}\right)_{2} \mathrm{C}_{6} \mathrm{H}_{3}\right)_{2}-2-(\mathrm{OH})-\mathrm{C}_{6} \mathrm{H}_{2}\right)\right\}_{2} \mathrm{l}(2 \mathrm{c})$}

Yield: $75 \%$. 'H NMR $\left(600 \mathrm{MHz}, \mathrm{CD}_{2} \mathrm{Cl}_{2}\right) \delta_{\mathrm{H}}(\mathrm{ppm}): 12.52(\mathrm{~s}$, $2 \mathrm{H}), 8.16(\mathrm{~s}, 2 \mathrm{H}), 8.05(\mathrm{~s}, 4 \mathrm{H}), 7.89(\mathrm{~s}, 16 \mathrm{H}), 7.87$ (br. s, $2 \mathrm{H}$, $\mathrm{O} H), 7.71(\mathrm{~s}, 4 \mathrm{H}), 7.70\left(\mathrm{~d},{ }^{4} J_{\mathrm{HH}}=2.1,2 \mathrm{H}\right), 7.24\left(\mathrm{~d},{ }^{4} J_{\mathrm{HH}}=2.1\right.$, $2 \mathrm{H}$ ). ${ }^{13} \mathrm{C}$ NMR $\left(151 \mathrm{MHz}, \mathrm{CD}_{2} \mathrm{Cl}_{2}\right.$ ) $\delta_{\mathrm{C}}$ (ppm): $170.46,159.01$, $146.87,141.76,140.25,138.89,134.37,133.28,133.25,132.73(\mathrm{q}$, $\left.{ }^{2} J_{\mathrm{CF}}=33\right), 132.68\left(\mathrm{q},{ }^{2} J_{\mathrm{CF}}=33\right), 132.27,132.04\left(\mathrm{q},{ }^{2} J_{\mathrm{CF}}=33\right)$, $131.98,130.74,130.61,130.14,128.85,127.37,124.04\left(\mathrm{q},{ }^{1} J_{\mathrm{CF}}=\right.$ $273), 123.91\left(\mathrm{q},{ }^{\prime} J_{\mathrm{CF}}=273\right), 123.62\left(\mathrm{q},{ }^{\prime} J_{\mathrm{CF}}=273\right), 122.59,122.16$, 121.74, 119.16. MALDI-TOF-MS: $m / z$ 2240.2 $\left([\mathrm{M}+\mathrm{H}]^{+}\right)$. Anal. calcd for $\mathrm{C}_{93} \mathrm{H}_{36} \mathrm{~F}_{54} \mathrm{~N}_{2} \mathrm{O}_{2}\left(M=2239.2 \mathrm{~g} \mathrm{~mol}^{-1}\right)$; $\mathrm{C} 49.88, \mathrm{H} 1.62, \mathrm{~F}$ 45.82 , N 1.25. Found: C 49.85, H 2.26, F 45.84, N 1.20

\section{General procedure for the synthesis of $\left(\kappa^{2}-N, O\right)$-salicylaldiminato nickel methyl pyridine complexes}

$5 \mathrm{~mL}$ of cold diethylether were added to a mixture of 3 equiv. of $\left[(\text { pyridine })_{2} \mathrm{NiMe}_{2}\right]$ and the respective difunctional salicylaldimine in a $25 \mathrm{~mL}$ Schlenk tube under stirring. A slow evolution of methane was observed within 15 to $30 \mathrm{~min}$. The deep red mixture was stirred for another $120 \mathrm{~min}$ at $25^{\circ} \mathrm{C}$. The solvent was removed in vacuo. The residue was dissolved in a small amount of benzene and filtered through a syringe filter. The volatiles were removed by sublimation at $-10^{\circ} \mathrm{C}$ (crushed ice/sodium chloride) under 
high vacuum $\left(10^{-3} \mathrm{mbar}\right)$ to yield quantitatively pure pyridine complexes.

\section{$I\left\{\mid\left(2,6-\left(3,5-\left(\mathrm{CF}_{3}\right)_{2} \mathrm{C}_{6} \mathrm{H}_{3}\right)_{2}-4-\mathrm{y} \mid-\mathrm{C}_{6} \mathrm{H}_{2}\right)-\mathrm{N}=\mathrm{C}(\mathrm{H})-(3,5-(3,5-\right.$} $\left.\left.\left(\mathrm{CF}_{3}\right)_{2} \mathrm{C}_{6} \mathrm{H}_{3}\right)_{2}-2-\mathrm{O}-\mathrm{C}_{6} \mathrm{H}_{2}\right)-\mathrm{K}^{2}-\mathrm{N}, \mathrm{O} \mid \mathrm{Ni}\left(\mathrm{CH}_{3}\right)($ pyridine $\left.\left.)\right\}_{2}\right]$ (3a)

Yield: 93\%. 'H NMR (400 MHz, $\left.\mathrm{CD}_{2} \mathrm{Cl}_{2}\right) \delta_{\mathrm{H}}(\mathrm{ppm}): 8.22(\mathrm{~s}, 8 \mathrm{H})$, 7.90 (br. s, $4 \mathrm{H}$ ), 7.82 (s, $4 \mathrm{H}), 7.72(\mathrm{~s}, 6 \mathrm{H}), 7.60$ (s, $2 \mathrm{H}), 7.57$, (s, $4 \mathrm{H}), 7.07$ (s, $2 \mathrm{H}), 6.95(\mathrm{~s}, 4 \mathrm{H}), 6.90(\mathrm{~s}, 2 \mathrm{H}), 6.72$, (s, $2 \mathrm{H})$, 6.58 (br. s, $2 \mathrm{H}$ ), 6.15 (br. s, $4 \mathrm{H}$ ), $-0.89(\mathrm{~s}, 6 \mathrm{H})$. Anal. calcd for $\mathrm{C}_{102} \mathrm{H}_{50} \mathrm{~F}_{48} \mathrm{~N}_{4} \mathrm{Ni}_{2} \mathrm{O}_{2}\left(M=2392.82 \mathrm{~g} \mathrm{~mol}^{-1}\right): \mathrm{C} 51.20, \mathrm{H} 2.12, \mathrm{~N}$ 2.34. Found: C $51.89, \mathrm{H} 2.29, \mathrm{~N} 2.17$

\section{$\mid \mathrm{CH}_{2}\left\{\mid\left(2,6-\left(3,5-\left(\mathrm{CF}_{3}\right)_{2} \mathrm{C}_{6} \mathrm{H}_{3}\right) 2-4-\mathrm{y} \mid-\mathrm{C}_{6} \mathrm{H}_{2}\right)-\mathrm{N}=\mathrm{C}(\mathrm{H})-(3,5-(3,5-\right.$ $\left.\left.\left(\mathrm{CF}_{3}\right)_{2} \mathrm{C}_{6} \mathrm{H}_{3}\right)_{2}-2-\mathrm{O}-\mathrm{C}_{6} \mathrm{H}_{2}\right)-\kappa^{2}-\mathrm{N}, \mathrm{O} \mid \mathrm{Ni}\left(\mathrm{CH}_{3}\right)($ pyridine $\left.\left.)\right\}_{2}\right](3 \mathrm{~b})$}

Yield: $89 \%$. 'H NMR (400 MHz, $\left.\mathrm{CD}_{2} \mathrm{Cl}_{2}\right) \delta_{\mathrm{H}}(\mathrm{ppm}): 8.32(\mathrm{~s}, 8 \mathrm{H})$, $8.08(\mathrm{~m}, 4 \mathrm{H}), 8.03(\mathrm{~s}, 4 \mathrm{H}), 7.87(\mathrm{~s}, 4 \mathrm{H}), 7.75(\mathrm{~s}, 4 \mathrm{H}), 7.69(\mathrm{~s}$, $4 \mathrm{H}), 7.61(\mathrm{~s}, 2 \mathrm{H}), 7.57(\mathrm{~s}, 2 \mathrm{H}), 7.51(\mathrm{~m}, 2 \mathrm{H}), 7.44(\mathrm{~s}, 4 \mathrm{H}), 7.14$ $(\mathrm{s}, 2 \mathrm{H}), 6.92(\mathrm{~m}, 4 \mathrm{H}), 4.30(\mathrm{~s}, 2 \mathrm{H}),-1.07(\mathrm{~s}, 6 \mathrm{H}) .{ }^{13} \mathrm{C}$ NMR (101 $\left.\mathrm{MHz}, \mathrm{CD}_{2} \mathrm{Cl}_{2}\right) \delta_{\mathrm{c}}(\mathrm{ppm}): 168.83,165.04,150.97,149.19$, $142.77,141.70,141.40,139.91,137.49,134.12,133.68,133.41$, $132.24\left(\mathrm{q},{ }^{\prime} J_{\mathrm{CF}}=273\right), 132.38\left(\mathrm{q},{ }^{1} J_{\mathrm{CF}}=273\right), 131.97,131.14$, $131.13,130.90\left(\mathrm{q},{ }^{1} J_{\mathrm{CF}}=273\right), 129.58,126.42,126.40,124.21$, $124.14,124.11\left(\mathrm{q},{ }^{2} J_{\mathrm{CF}}=33\right), 124.09\left(\mathrm{q},{ }^{2} J_{\mathrm{CF}}=33\right), 124.06(\mathrm{q}$, $\left.{ }^{2} J_{\text {CF }}=33\right), 122.05,122.01,121.01,120.57,120.25,40.88,-7.37$. Anal, calcd for $\mathrm{C}_{103} \mathrm{H}_{52} \mathrm{~F}_{48} \mathrm{~N}_{4} \mathrm{Ni}_{2} \mathrm{O}_{2}\left(M=2406,85 \mathrm{~g} \mathrm{~mol}^{-1}\right)$ : $\mathrm{C} 51.40$, H 2.18, N 2.18. Found: C 51.86, H 2.26, N 2.12.

\section{IC(CF $)_{2}\left\{\mid\left(2,6-\left(3,5-\left(\mathrm{CF}_{3}\right)_{2} \mathrm{C}_{6} \mathrm{H}_{3}\right) 2-4-\mathrm{yl}-\mathrm{C}_{6} \mathrm{H}_{2}\right)-\mathrm{N}=\mathrm{C}(\mathrm{H})-(3,5-(3,5-\right.$} $\left.\left(\mathrm{CF}_{3}\right)_{2} \mathrm{C}_{6} \mathrm{H}_{3}\right)$ 2-2-O-C $\left.6 \mathrm{H}_{2}\right)-\mathrm{K}^{2}-\mathrm{N}, \mathrm{O}$ ONi( $\left.\mathrm{CH}_{3}\right)$ (pyridine) $\left.\}_{2}\right](3 \mathrm{c})$

Yield: 77\%, 'H NMR (400 MHz, $\left.\mathrm{CD}_{2} \mathrm{Cl}_{2}\right) \delta_{\mathrm{H}}(\mathrm{ppm}): 8.26(\mathrm{~s}, 8 \mathrm{H})$, $8.08\left(\mathrm{dt},{ }^{3} J_{\mathrm{HH}}=5.1,4 \mathrm{H}\right.$, meta-pyr $), 8.06(\mathrm{~s}, 4 \mathrm{H}), 7.89(\mathrm{~s}, 4 \mathrm{H})$, $7.78(\mathrm{~s}, 4 \mathrm{H}), 7.68(\mathrm{~s}, 4 \mathrm{H}), 7.65(\mathrm{~s}, 4 \mathrm{H}), 7.62(\mathrm{~s}, 2 \mathrm{H}), 7.60(\mathrm{~d}$, $\left.{ }^{4} J_{\mathrm{HH}}=2.5,2 \mathrm{H}\right), 7.54\left(\mathrm{t},{ }^{3} J_{\mathrm{HH}}=7.7,2 \mathrm{H}\right), 7.18\left(\mathrm{~d},{ }^{4} J_{\mathrm{HH}}=2.5\right.$, $2 \mathrm{H}), 6.94\left(\mathrm{t},{ }^{3} J_{\mathrm{HH}}=7.7,4 \mathrm{H}\right.$, ortho-pyr $),-1.12(\mathrm{~s}, 6 \mathrm{H}) \cdot{ }^{13} \mathrm{C} \mathrm{NMR}$ $\left(101 \mathrm{MHz}, \mathrm{CD}_{2} \mathrm{Cl}_{2}\right) \delta_{\mathrm{C}}(\mathrm{ppm}): 168.54,165.26,151.63,150.96$, $142.66,141.24,140.68,137.66,134.59,134.10,133.39,132.97$, $132.55\left(\mathrm{q},{ }^{2} J_{\mathrm{CF}}=33\right), 132.47\left(\mathrm{q},{ }^{2} J_{\mathrm{CF}}=33\right), 132.39,132.25,131.04$, $131.02,130.96\left(\mathrm{q}^{2} J_{\mathrm{CF}}=33\right), 129.60,129.58,126.47,126.44$, $124.59,124.25,124.11\left(\mathrm{q},{ }^{1} J_{\mathrm{CF}}=273\right), 124.04\left(\mathrm{q},{ }^{1} J_{\mathrm{CF}}=273\right)$, $123.91\left(\mathrm{q},{ }^{1} J_{\mathrm{CF}}=273\right), 122.70,120.77,120.69,120.41,-7.68$. Anal. calcd for $\mathrm{C}_{105} \mathrm{H}_{50} \mathrm{~F}_{54} \mathrm{~N}_{4} \mathrm{Ni}_{2} \mathrm{O}_{2}\left(M=2540.18 \mathrm{~g} \mathrm{~mol}^{-1}\right)$ : $\mathrm{C} 49.59, \mathrm{H}$ 1.98, F 40.35, N 2.20. Found: C 49.74, H 2.02, F 40.32, N 2.11 .

\section{Polymerizations in organic solvent}

The polymerizations in organic solvent were carried out in a $250 \mathrm{~mL}$ stainless steel mechanically stirred $(750 \mathrm{rpm})$ pressure reactor equipped with a heating/cooling jacket supplied by a thermostat controlled by a thermocouple dipping into the polymerization mixture. A valve controlled by a pressure transducer allowed for applying and keeping up a constant ethylene pressure. The required flow of ethylene, corresponding to ethylene consumed by polymerization, was monitored by a mass flow meter and recorded digitally. Prior to a polymerization experiment, the reactor was heated under vacuum to the desired reaction temperature for 30-60 min, back-filled with argon, and charged with solvent. The catalyst precursor was weighed into a dry syringe in the glovebox. In a slight argon stream, solvent from the reaction was pulled into the syringe several times, dissolving and transferring to the reactor the catalyst precursor. The reactor was closed and a constant ethylene pressure was applied. After the desired reaction time, the reactor was rapidly vented and cooled to room temperature. The reaction mixture was stirred with an excess volume of methanol. The polymer was isolated by filtration, washed several times with methanol, and dried in vacuo at $50^{\circ} \mathrm{C}$

\section{Polymerizations in supercritical carbon dioxide $\left(\mathrm{scCO}_{2}\right)$}

The polymerizations in $\mathrm{scCO}_{2}$ were carried out in a high-pressure view cell (NWA GmbH, Lörrach, Germany). The stainless steel cell has a variable internal volume between 30 and $60 \mathrm{~cm}^{3}$ tuneable by means of a piston operated by a hydraulic system. The piston material is a transparent sapphire which, together with a sapphire view window on the opposite side of the cell, allows for visually observing the contents of the cell. The cell contents can be agitated by means of a magnetically coupled stainless steel mechanical propeller stirrer. Heating is provided by means of two stainless steel cartridge heaters lodged in cavities of the metallic body of the cell. The temperature inside the cell is measured by a thermocouple, and the pressure is monitored with a Bourdon type manometer.

The gases were introduced via high pressure pumps (up to 60 $\mathrm{MPa}$ for carbon dioxide and up to $40 \mathrm{MPa}$ for ethylene) which employ the corresponding cold condensed liquids as a reservoir. The rate of the ethylene addition was controlled by a two way HPLC valve, with an internal loop volume of $1 \mathrm{~mL}$. The quantities of gases introduced were estimated by the change in piston position and the pressure. Prior to a polymerization experiment, the reactor was heated under a low pressure of carbon dioxide $(6 \mathrm{MPa})$ to the desired reaction temperature for 30 to $60 \mathrm{~min}$, and then flushed 3 times with carbon dioxide. The catalyst precursor was added as a compressed pellet via a modified syringe to the empty reactor. The reactor was closed and filled with carbon dioxide to the desired pressure $(10 \mathrm{MPa})$. Under continuous stirring the pressure was increased (to $30 \mathrm{MPa}$ ) by decreasing the cell volume via the piston, and the ethylene was added at constant pressure. After the addition of the desired amount of monomer, polymerizations was carried out at a pressure of $65 \mathrm{MPa}$, adjusted by moving the piston forward. The reaction was stopped by increasing the volume of the cell and the reactor was carefully vented.

\section{Acknowledgements}

Financial support by the BMBF (project $03 \times 5505$ ) is gratefully acknowledged. S. M. is indebted to the Fonds der Chemischen Industrie

\section{References}

I (a) S. D. Ittel, L. K. Johnson and M. Brookhart, Chem. Rev., 2000 100, 1I69-1203; (b) V. C. Gibson and S. K. Spitzmesser, Chem. Rev., 2003, 103, 283-316; (c) S. Mecking, Angew. Chem., Int. Ed., 2001, 40, 534-540; (d S) S. Mecking, Coord Chem. Rev, 2000, 203, 325-351; (e) Z. Guan, Chem-Eur. J., 2002, 8, 3086-3092; (f) J. Durand and B. Milani, Coord. Chem. Rev., 2006, 250, 542-560; (g) G. J. Domski, J. M. Rose, G. M. Coates, A. D. Bolig and M. Brookhart, Prog. Polym. Sci., 2007, $32,30-92$.

2 (a) L. S. Boffa and B. M. Novak, Chem. Rev, 2000, 100, 1479-1493; (b) A. Berkefeld and S. Mecking, Angew. Chem., Int. Ed., 2008, 47 
$2538-2 \$ 42 ;(c)$ S. Mecking, A. Held and F. M. Bauers, Angew. Chem., Int. Ed., 2002, 41, 544-561.

3 (a) L. K. Johnson, S. Mecking and M. Brookhart, J. Am. Chem. Soc, $1996,118,267-268$; (b) S. Mecking, L. K. Johnson, L. Wang and M. Brookhart, J. Am. Chem. Soc, 1998, 120, 888 899; (c) L. Johnson, L. Wang, S. McLain, A. Bennett, K. Dobbs, E. Hauptman, A. Ionkin, S. Ittel, K. Kunitsky, W. Marshall, E. McCord, C. Radzewich, A. Rinehart, K. J. Sweetman, Y. Wang, Z. Yin and M. Brookhart, ACS Symp. Ser, 2003, 857, 131-142; (d) E. Drent, R. van Dijk, R. van Ginkel, B. van Oort and R. I. Pugh, Chem. Commum., 2002, 744 745; (e) K. M. Skupov, P. R. Marella, M. Simard, G. P. A. Yap, N. Allen, D. Conner, B. L. Goodall and J. P. Claverie, Macromol, Rapid Commun., $2007,28,2033-2038$.

4 T. Kochi, S. Noda, K. Yoshimura and K. Nozaki, J. Am. Chem. Soc, $2007,129,8948-8949$.

5 D. Guironnet, P. Roesle, T. Rünzi, I. Göttker-Schnetmann and S. Mecking, J. Am. Chem. Soc, 2009, 131, 422-423.

6 Also cf(a) W. Weng, Z. Shen and R. F. Jordan, J. Am. Chem. Soc, 2007, $129,15450-15451 ;(b)$ C. Chen, S. Luo and R. F. Jordan, J. Am. Chem. Soc., 2008, 130, 12892-12894: (c) K. M. Skupov, L. Piche and J. P. Claverie, Macromolecules, 2008, 41, 2309-2310; (d) S. Liu, S. Borkar, D. Newsham, H. Yennawar and A. Sen, Organometallics, 2007, 26, 210-216.

7 (a) A. Held, F. M. Bauers and S. Mecking, Chem. Commun., 2000, 301302; (b) A. Tomov, J.-P. Broyer and R. Spitz, Macromol. Symp., 2000, 150, 53-58; (c) R. Soula, C. Novat, A. Tomov, R. Spitz, J. Claverie, X. Drujon, J. Malinge and T. Saudemont, Macromolecules, 2001, 34, 2022-2026; (d) I. Göttker-Schnetmann, B. Korthals and S. Mecking, J. Am. Chem. Soc., 2006, 128, 7708-7709; (e) S.-M. Yu and S. Mecking, Macromolecules, 2009, 42, 3669-3673.

8 (a) L. K. Johnson, C. M. Killian, S. D. Arthur, J. Feldman, E. F, McCord, S. J. McLain, K. A. Kreutzer, M. A. Bennett, E. B. Coughlin, S. D. Ittel, A. Parthasarathy, D. J. Tempel, M. S. Brookhart (DuPont, Univ. North Carolina) wo 96/23010, 1996; (b) T. J. De Vries, R. Duchateau, M. A. G. Vorstman and J. T. F. Keurentjes, Chem. Commun., 2000, 263-264; (c) T. J. De Vries, M. F. Kemmere and J. T. F. Keurentjes, Macromolecules, 2004, 37, 4241-4246; (d) A. Bastero, G. Franciò, W. Leitner and S. Mecking, Chem.-Eur. J., 2006, 12, 6110-6116; (e) D. Guironnet, I. Göttker-Schnetmann and S. Mecking, Macromolecules, 2009, DOI: 10.1021/ma901397q.

9 (a) Chemical Synthesis Using Supercritical Fluids ed. P. G. Jessop and W. Leitner, Wiley-VCH, Weinheim, 1999; (b) Supercritical Carbon Dioxide in Polymer Reaction Engineering ed. M. F. Kemmere and T. Meyer, Wiley-VCH, Weinheim, 2005.

10 (a) J. L. Kendall, D. A. Canelas, J. L. Young and J. M. DeSimone, Chem. Rev, 1999, 99, 543-563; (b) S. L. Wells and J. DeSimone, Angew, Chem., Int. Ed., 2001, 40, 518-527.

11 (a) J. M. DeSimone, E. E. Maury, Y. Z. Menceloglu, J. B. McClain, T. J. Romack and J. R. Combes, Science, 1994, 265, 356-359; (b) S. Beuermann, M. Buback, C. Schmaltz and F. D. Kuchta, Macromol. Chem. Phys, 1998, 199, 1209-1216; (c) A. I. Cooper, W, P. Hems and A. B. Holmes, Macromol. Rapid Commun., 1998, 19, 353-357; (d) U. Beginn, R. Najjar, J. Ellmann, R. Vinokur, R. Martin and M. Moeller, J. Polym. Sci., Part A: Polym. Chem., 2006, 44, 1299-1316; (e) A. M. Gregory, K. J. Thurecht and S. M. Howdle, Macromolecules, 2008, 41, $1215-1222$

12 Ring opening metathesis polymerization: (a) A. Fürstner, L. Ackermann, K. Beck, H. Hori, D. Koch, K. Langemann, M. Liebl, C. Six and W. Leitner, J. Am. Chem. Soc, 2001, 123, 9000 9006; (b) A. Fürstner, D. Koch, K. Langemann, W. Leitner and C. Six, Angew. Chem., Int. Ed. Engl, 1997, 36, 2466-2469; (c) C. D. Mistele, H, H. Thorp and J. M. DeSimone, J. Macromol. Sci, Part A: Pure Appl. Chem., 1996, $33,953-960$.
13 Polycarbonate synthesis from $\mathrm{CO}_{2}$ and epoxide: (a) D. J. Darensbourg, N. W. Stafford and T. Katsurao, J. Mol. Catal. A: Chem, 1995, 104, L1 L4; (b) C. A. Costello, E. Berluche, S. J. Han, D. A. Sysyn, M. S. Super and E. J. Beckman, PMSE preprints, 1996, 74, 430; (c) M. Duper, E. Berluche, C. Costello and E. Beckman, Macromolecules, 1997, 30, 368-372.

14 Phenyl acetylene polymerization: H. Hori, C. Six and W. Leitner, Macromolecules, 1999, 32, 3178-3182.

15 Early work: (a) W. Keim, F. H. Kowaldt, R. Goddard and C Krüger, Angew. Chem., Int. Ed. Engl., 1978, 17, 466 467; (b) K. A. OstojaStarzewski and J. Witte, Angew. Chem. Int. Ed. Engl., 1987, 26, 63-64; (c) U. Klabunde and S. D. Ittel, J. Mol. Catal., 1987, 41, 123-134.

16 (a) C. Wang, S. Friedrich, T. R. Younkin, R. T. Li, R. H. Grubbs, D. A. Bansleben and M. W. Day, Organometallics, 1998, 17, 3149 3151; (b) L. K. Johnson, A. M. A. Bennett, S. D. Ittel, L. Wang, A. Parthasarathy, E. Hauptman, R. D. Simpson, J. Feldman, E. B. Coughlin, (DuPont) WO98/30609, 1998; (c) T. R. Younkin, E. F. Connor, J. I. Henderson, S. K. Friedrich, R. H. Grubbs and D. A Bansleben, Science, 2000, 287, 460-462; $(d)$ F. A. Hicks and M. Brookhart, Organometallics, 2001, 20, 3217-3219; (e) R. Soula, J. P. Broyer, M. F. Llauro, A. Tomov, R. Spitz, J. Claverie, X. Drujon, J. Malinge and T. Saudemont, Macromolecules, 2001, 34, 2438-2442; (f) V. C. Gibson, A. Tomov, A. J. P. White and D. J. Williams, Chem. Commun., 2001, 719-720; (g) M. Zuideveld, P. Wehrmann, C. Röhr and S. Mecking, Angew. Chem., Int. Ed., 2004, 43, 869-873; (h) J. C. Jenkins and M. Brookhart, J. Am. Chem. Soc, 2004, 126, 58275842; (i) L. Zhang, M. Brookhart and P. S. White, Organometallics, 2006, 25, 1868-1874; (j) P. Kuhn, D. Sémeril, C. Jeunesse, D. Matt, M. Neuburger and A. Mota, Chem.-Eur, J., 2006, 12, 5210-5219; (k) I. Göttker-Schnetmann, P. Wehrmann, C. Röhr and S. Mecking, Organometallics, 2007, 26, 2348-2362; (l) S.-M. Yu, A. Berkefeld, 1. Göttker-Schnetmann, G. Müller and S. Mecking, Macromolecules, 2007, 40, 421 428; (m) A, Bastero, I. Göttker-Schnetmann, C. Röhr and S. Mecking, Adv. Synth Catal, 2007, 349, 2307-2316; (n) J. Pietsch, P. Braunstein and Y. Chauvin, New J. Chem., 1998, 22, 467-472; (o) F. Speiser, P. Braunstein and L. Saussine, Acc. Chem. Res, 2005, 38, 784-793; (p) D. Guironnet, T. Rünzi, I. Göttker-Schnetmann and S. Mecking, Chem. Commun., 2008, 4965-4967; (q) Y. Schneider, J. D. Azoulay, R. C. Coffin and G. C. Bazan, J. Am. Chem. Soc., 2008, 130, $10464-10465$

17 (a) A. Berkefeld and S. Mecking, $J$. Am. Chem. Soc, 2009, 131, 15651574; (b) A. Berkefeld, M. Drexler, H. Möler and S. Mecking, J. Am. Chem. Soc., 2009, 131, 12613-12622.

18 (a) K. Kurtev and A. Tomov, J. Mol. Catal., 1994, 88, 141 150; (b) D. Zhang and G.-X Jin, Organometallics, 2003, 22, 2851-2854; (c) D. Zhang and G.-X Jin, Inorg. Chem. Commun., 2006, 9, 1322-1325; $(d) \mathrm{T}$. $\mathrm{Hu}, \mathrm{L} .-\mathrm{M}$. Tang, X.-F. Li, Y.-S. Li and N.-H. Hu, Organometallics, $2005,24,2628-2632$; (e) S. S, D. J. Joe, S. J. Na, Y.W. Park, C. H Choi and B. Y. Lee, Macromolecules, 2005, 38, 10027-10033; (f) P. Wehrmann and S. Mecking, Organometallics, 2008, 27, 1399-1408; (g) B. A. Rodriguez, M. Delferro and T. J. Marks, Organometallics, 2008, 27, 2166-2168; (h) B. A. Rodriguez, M. Delferro and T. J. Marks, J. Am. Chem. Soc., 2009, 131, 5902-5919.

19 E. F. Connor, T. R. Younkin, J. 1. Henderson, A. W. Waltman and R. H. Grubbs, Chem. Commun., 2003, 2272-2273.

20 (a) L. K. Johnson, M. C. Killian and M. Brookhart, J. Am. Chem. Soc., $1995,117,6414-6415$; (b) Z. Guan. P. M. Cotts, E. F. McCord and S. J. McLain, Science, 1999, 283, 2059-2062; (c) Also cf.: V. M. Mochring and G. Fink, Angew. Chem., Int. Ed. Engl., 1985, 24, 1001-1003.

21 J. Càmpora, M.d.M. Conejo, K. Mereiter, P. Palma, C. Pérez, M. L. Reyes and C. Ruiz, J. Organomet. Chem., 2003, 683, $220-239$.

22 P. Wehrmann, M. Zuideveld, R. Thomann and S. Mecking, Macromolecules, 2006, 39, 5995-6002. 